\title{
A Standardized Tank Design for the Light Dark Task in Zebrafish Amanda Facciol ${ }^{1, \#, *}$, Steven Tran ${ }^{2, \#}$ and Robert Gerlai ${ }^{1,3}$
}

${ }^{1}$ Department of Cell and Systems Biology, University of Toronto, Mississauga, Canada; ${ }^{2}$ Department of Biology and Biological Engineering, California Institute of Technology, Pasadena, USA; ${ }^{3}$ Department of Psychology, University of Toronto, Mississauga, Canada

*For correspondence: amanda.facciol@mail.utoronto.ca

\#Contributed equally to this work

[Abstract] The light dark paradigm is a common behavioral test used to screen a variety of pharmacological agents, including anxiogenics and anxiolytics. Although most often used in rodents, the light dark task has recently been adapted for use in zebrafish. However, a number of inconsistent findings have been reported for this species. Some have found zebrafish to prefer black, while others report a preference for light. Careful analysis of light dark preference experiments using zebrafish reveals significant variation in testing tank design and test conditions, including lighting and substrate color. Additionally, in some experiments the designated dark side of the testing tank is completely covered, producing a "cave-like" environment which further confounds results. Lastly, authors commonly use the terms "light vs. dark" interchangeably with "white vs. black", when these are two separate factors that may influence preference: illumination level vs. background shade. To address these limitations, we designed testing tanks that differentiate illumination vs. background shade preference in zebrafish. This design allows for simple standardization of light dark testing apparatus in zebrafish, and facilitates more reliable comparison across studies.

Keywords: Zebrafish, Anxiety, Background shade, Illumination, Light dark task

[Background]The light dark paradigm relies on rodents' natural avoidance for open, well-illuminated areas, a behavior also called scototaxis (Champagne et al., 2010). Therefore, in the light dark task, rodents often spend more time in the dark compartment (Champagne et al., 2010; Hascoet et al., 2001). Alteration of this natural scototaxic response allows researchers to investigate the anxiogenic and anxiolytic properties of certain pharmacological agents (Sousa et al., 2006; Hascoet et al., 2001). While this paradigm has recently been adapted for use in zebrafish, the transference of this task from rodents to zebrafish has resulted in a number of inconsistencies. Unlike the nocturnal rodent, zebrafish are diurnal which may pose an issue for generalizing scototaxic and phototaxic responses across these species.

Although the light dark task has been used for a number of years in zebrafish, findings of zebrafish light dark preference remain inconsistent. Some studies have found zebrafish to prefer the black compartment (Serra et al., 1999; Maximino et al., 2010; Blaser and Peñalosa, 2011; Facciol et al., 2017), whereas others find preference for the light compartment (Gerlai et al., 2000; Champagne et al., 2010; Blaser and Peñalosa, 2011). In a study by Facciol et al. (2017), we suggested these inconsistencies 
may be due to a number of factors. Firstly, literature often confuses illumination level and background shade, using the term "light" interchangeably with "white", and "dark" with "black". Additionally, light-dark studies in zebrafish have seen a significant variation in testing tank apparatus. Factors such as the use of substrate (i.e., sand or gravel), type and levels of lighting (e.g., ambient lighting vs. tank lighting), and presence of dividers between compartments have not been kept constant across studies. These inconsistencies make it difficult to compare results across studies and may contribute to the discordant findings obtained in recent years. If these inconsistencies are indeed due to variation in testing design, standardization of testing methods is highly important for correct interpretation of pharmacological studies. The purpose of this protocol is to outline the construction and implementation of a standardized testing design for the light-dark task in zebrafish. We hope that this standardization will allow for better cross study comparison and proper interpretation of results of analyses of pharmacological agents obtained with the light-dark paradigm.

\section{Materials and Reagents}

1. Zebrafish

2. Ethanol ( $100 \%$ ethyl alcohol anhydrous, Commercial Alcohols)

\section{Equipment}

1. Four $1.5 \mathrm{~L}$ tanks for pre-exposure (Aquaneering, catalog number: ZT140, Figure 1)

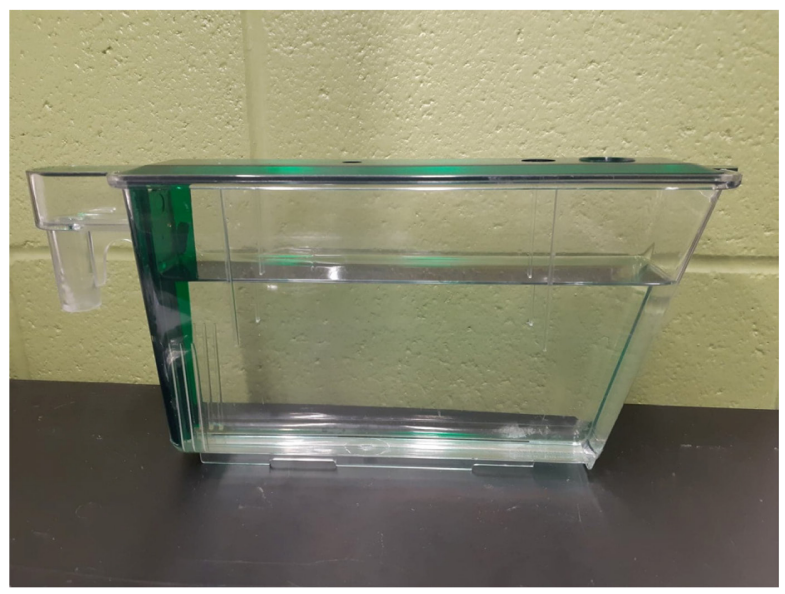

Figure 1. Pre-exposure tanks

2. Two larger tank lights for above pre-exposure tanks (Aqueon fluorescent light strip, 36-inch, 15 watt)

3. Fish nets (Underwater Treasures, catalog number: 4473)

Note: It is important to have two different sets of nets for control and ethanol tanks, to avoid ethanol cross-contamination. 
4. Four small, clear, plastic holding containers (one for each testing tank, Figure 2) (Walmart, UPC 7169149736 , or something of equivalent size)

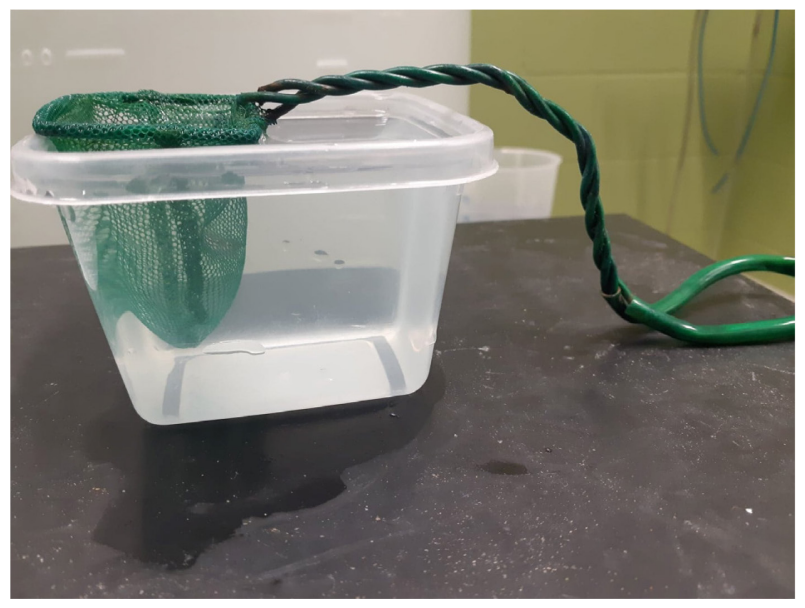

Figure 2. Holding containers

5. Four cameras for behavioral recording (JVC Everio GZ-MG750RU)

6. Four $40 \mathrm{~L}$ glass tanks $(50 \mathrm{~cm} \times 30 \mathrm{~cm} \times 25.5 \mathrm{~cm})$

7. Four aquarium light fixtures (Aqueon fluorescent light strip, 18-inch, 15 watt)

8. White and black corrugated plastic sheets (Home Depot, SKU 1000171720)

9. Ruler

10. Pencil/pen

11. Scissors/exactoknife

12. Aquarium safe silicone (Aqueon, catalog number: 12888)

\section{Procedure}

A. Tank construction

The following outlines the construction of all 4 tanks as seen in Figure 3 and Figure 4 (i.e., Tank 1: all white, half illuminated; Tank 2: all black, half illuminated; Tank 3: half white and half black, low illumination throughout; Tank 4: half white and half black, high illumination throughout).

1. Ensure all tanks are clean and dry.

2. Measure and cut all corrugated plastic pieces to fit internal dimensions of the tanks.

a. For Tanks 1 and 2, cut one bottom piece $(49 \mathrm{~cm} \times 24.5 \mathrm{~cm})$, one back piece $(49 \mathrm{~cm} \times$ $29 \mathrm{~cm}$ ) and two side pieces $(24.5 \mathrm{~cm} \times 29 \mathrm{~cm}$ ) per tank (total of 8 pieces, 4 black and 4 white).

b. For Tanks 3 and 4, cut two bottom $(24.5 \mathrm{~cm} \times 24.5 \mathrm{~cm})$, two back $(24.5 \mathrm{~cm} \times 29 \mathrm{~cm})$ and two side $(24.5 \mathrm{~cm} \times 29 \mathrm{~cm})$ pieces per tank (total of 12 pieces, 6 black and 6 white).

3. Use the aquarium safe silicone to secure the corrugated plastic pieces in place. Silicone the bottom pieces first, so the side and back pieces can rest on them. 
Note: For Tanks 3 and 4, arrange so one tank has black on left and white on right, and the other has black on right and white on left, as seen in Figure 3.

4. Cut dividers $(24.5 \mathrm{~cm} \times 26 \mathrm{~cm})$ for each tank. For Tanks 1 and 2, cut one per tank. For Tanks 3 and 4 , cut 2 per tank (one black and one white).

5. Cut four holes in each divider ( 2 high and 2 low; $4 \mathrm{~cm} \times 4 \mathrm{~cm}$ ) spaced evenly (approximately $5 \mathrm{~cm}$ apart). Once all dividers are prepared, silicone dividers for Tanks 3 and 4 together, creating a white and black side on each.

6. Allow silicone to set for $24 \mathrm{~h}$. After this, fill the tanks with water for at least 2 days to allow for complete degassing of silicone.

7. Take four standard $40 \mathrm{~L}$ tank light fixtures, one per tank. To create the dark compartment in Tanks 1 and 2, cover half the overhead light with 4 sheets of standard printer paper (cut to $24 \mathrm{~cm} \times 11 \mathrm{~cm}$ ), creating a light and dark compartment. To create a full tank low illumination condition as in Tank 3, cover the entire light with 4 sheets of standard printer paper (cut to $48 \mathrm{~cm} \times 11 \mathrm{~cm}$ ). To create a full tank high illumination condition as in Tank 4, leave the light completely uncovered.

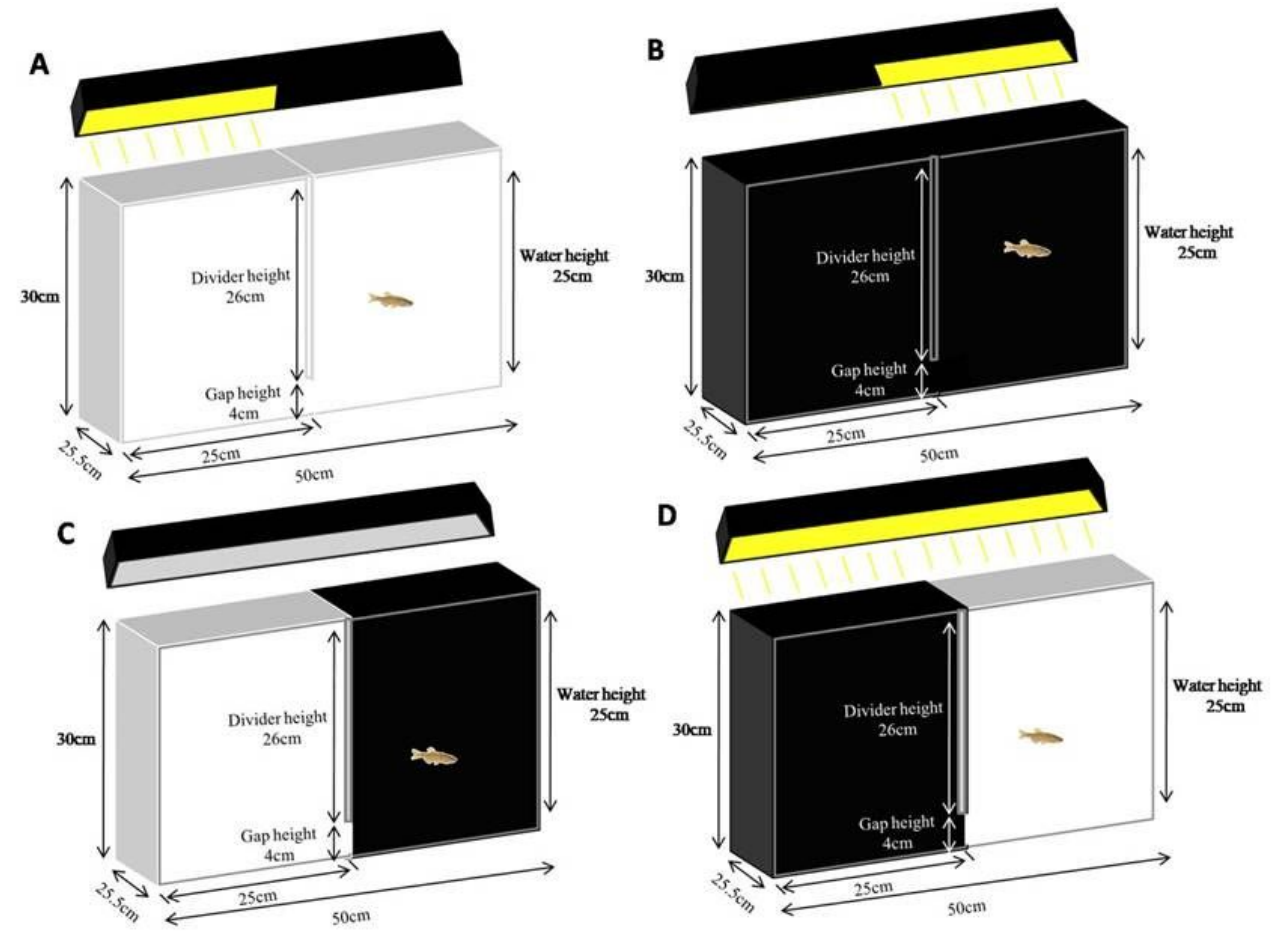

Figure 3. Schematic image of tank design (with dimensions) showing (A) all white tank, (B) all black tank, (C) low illumination tank, and (D) high illumination tank 


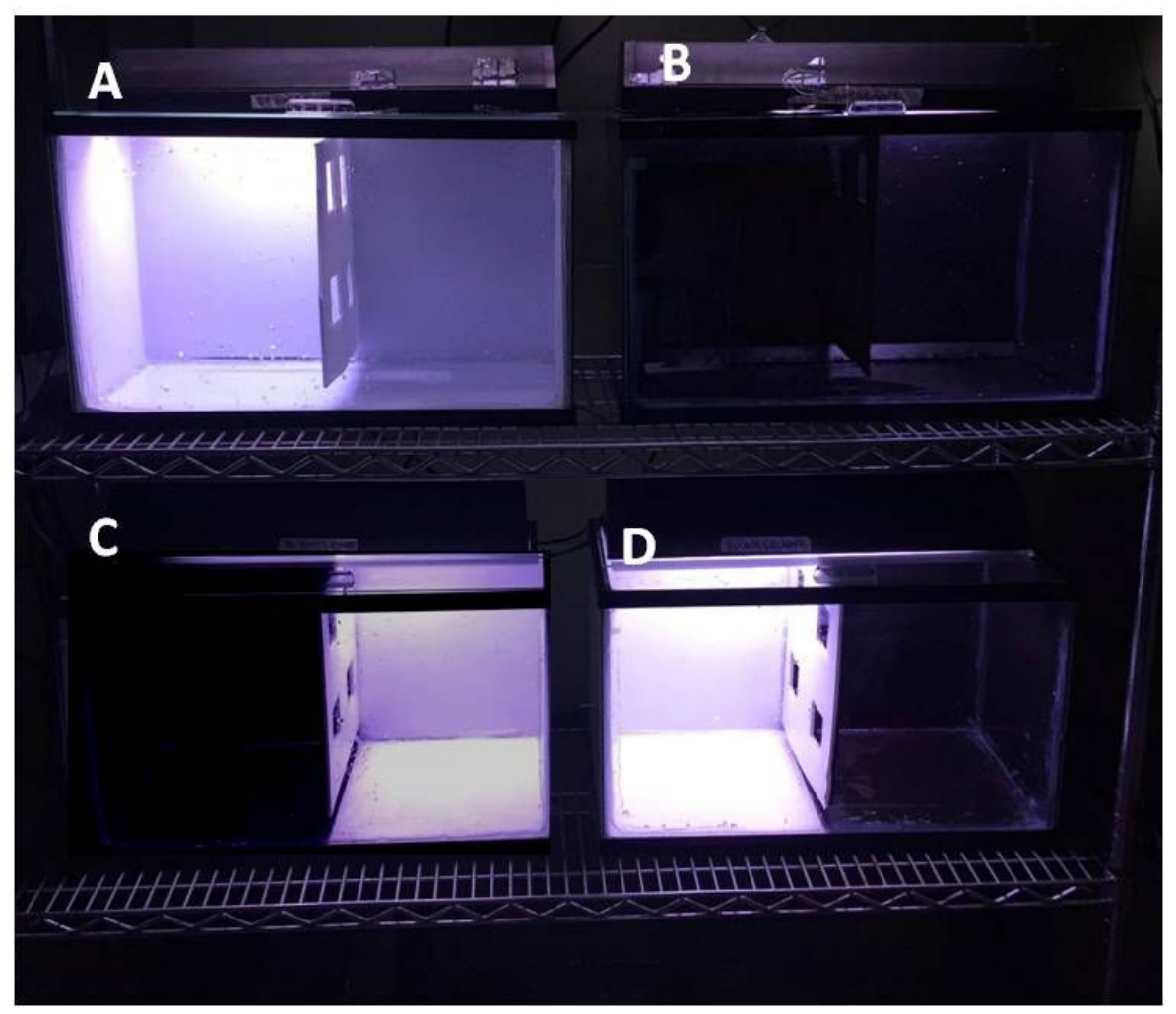

Figure 4. Photographs of actual experimental tanks showing (A) all white tank, (B) all black tank, (C) low illumination tank, and (D) high illumination tank

B. Method validation

Recent inconsistencies in zebrafish light dark literature may be due to a number of reasons. Firstly, terms such as "light vs. dark" are used interchangeably with "white vs. black", leading to inconsistent interpretation (Serra et al., 1999; Blaser and Peñalosa, 2011). Additionally, there is significant variance in testing apparatus, with inconsistencies in level and type of lighting, type and color/shade of substrate, and whether dividers between compartments are used (Serra et al., 1999; Gerlai et al., 2000; Maximino et al., 2007; Blaser and Peñalosa, 2011; Facciol et al., 2017). In studies that examine illumination preference (i.e., light vs. dark), it is common practice to cover one entire half of the tank to create the dark compartment (Gerlai et al., 2000; Champagne et al., 2010; Blaser and Peñalosa, 2011). However, this creates the confound of a "cave-like environment" rather than simply a dark area.

Previously in our laboratory, we dissociated the effects of level of illumination from that of background shade and thus demonstrated that these factors affect zebrafish behavior independently. For example, we found zebrafish to prefer black over white (Facciol et al., 2017), but exhibited no preferential responses to compartments with different levels of illumination. Following this, we decided to further validate the use of our methods through application of ethanol.

Details of fish maintenance, behavioral recording and quantification methods and statistical methods have been described elsewhere (Facciol et al., 2019). The following section will outline methods specific for the testing procedures. 


\section{Testing procedure}

1. Prepare pre-exposure, holding and testing tanks.

a. Fill four $40 \mathrm{~L}$ tanks to $25 \mathrm{~cm}$ high with water (approximately $30 \mathrm{~L}$ ).

b. Fill four $1.5 \mathrm{~L}$ tanks with $1 \mathrm{~L}$ of water.

c. Fill four holding containers with $500 \mathrm{ml}$ water.

Note: For control trials, leave tanks as is. For ethanol trials, create a 1\% (vol/vol) ethanol solution in all of the tanks. For the $40 \mathrm{~L}$ testing tanks, remove $300 \mathrm{ml}$ of water and replace with $300 \mathrm{ml}$ of ethanol. Similarly, in the $1.5 \mathrm{~L}$ tanks, remove $10 \mathrm{ml}$ of water and replace with $10 \mathrm{ml}$ ethanol. Lastly, in the holding containers, remove $5 \mathrm{ml}$ water and replace with $5 \mathrm{ml}$ ethanol.

2. Net fish one at a time and place in nets in holding containers, as seen in Figure 2.

3. Ensure ambient room lights are off (and remain off for entire testing procedure), testing tank lights are off and pre-exposure lights are on. Start cameras for behavioral recording (if recording pre-exposure) and place one fish into each pre-exposure tank for $30 \mathrm{~min}$.

4. After $30 \mathrm{~min}$, turn off cameras, remove fish and place back into holding tank.

5. Turn off pre-exposure lights and turn on testing tank lights.

6. Start cameras and place pre-exposed fish into testing tanks (one fish per tank) for another $30 \mathrm{~min}$.

Note: For Tanks 1 and 2, ensure the light and dark compartments are randomized between trials (by simply turning the tank light backward). For Tanks 3 and 4, ensure to randomize white and black sides i.e., tanks were randomized between low illumination with black on left, low illumination with black on right, high illumination with black on left, and high illumination with black on right.

7. After $30 \mathrm{~min}$, turn off cameras, remove fish from tanks and wash before returning back to housing tanks (if ethanol exposed).

8. Following video recording of behavioral responses in a light dark experiment, videos will be played back to videotracking software (ex. Ethovision XT) for behavioral quantification. Commonly investigated parameters include anxiety-related behaviors (i.e., freezing, bottom dwelling, erratic movement) and behaviors that suggest side preference (i.e., latency to enter light/dark or white/black side, total time spent in light/dark or white/black side) (Gerlai et al., 2000).

\section{Data analysis}

The detailed results of this study have been published elsewhere (Facciol et al., 2019). Briefly, zebrafish showed no illumination preference in the all-white tank, however illumination preference in the all-black tank was alcohol dependent in the first $5 \mathrm{~min}$, with control zebrafish exhibiting phototaxis and ethanol exposed zebrafish exhibiting scototaxis. In the background shade preference task, neither control nor ethanol exposed zebrafish exhibited a preference, regardless of illumination level. This lack of preference, which has also been shown elsewhere (Facciol et al., 2017), may 
simply be due to slight modification of the testing arena (i.e., addition of dividers), further reinforcing the need for standardized testing tanks to allow for experimental comparisons.

In the illumination preference task, the lack of preference in the all-white tank may be due to a compounding effect of both an aversive white background and high illumination, creating a highly anxiogenic environment. Our results suggest the combination of these two factors may produce an environment that negates the effect of ethanol, which could pose an issue for studies using the light dark task for pharmacological validation. Overall, our study highlights the need for further investigation of optimal parameters for both background shade and illumination in the light dark task.

\section{Acknowledgments}

These methods were adapted from a previous study (Facciol et al., 2017). This project was funded by an NSERC Discovery Grant awarded to Dr. Robert Gerlai (Grant\# 311637).

\section{Ethics}

All protocols approved by the Animal Care Committee (ACC) at the University of Toronto (Protocol\# 20011370).

\section{$\underline{\text { References }}$}

1. Blaser, R. E. and Peñalosa, Y. M. (2011). Stimuli affecting zebrafish (Danio rerio) behavior in the light/dark preference test. Physiol Behav 104(5): 831-837.

2. Champagne, D. L., Hoefnagels, C. C., de Kloet, R. E. and Richardson, M. K. (2010). Translating rodent behavioral repertoire to zebrafish (Danio rerio): relevance for stress research. Behav Brain Res 214(2): 332-342.

3. Facciol, A., Iqbal, M., Eada, A., Tran, S. and Gerlai, R. (2019). The light-dark task in zebrafish confuses two distinct factors: Interaction between background shade and illumination level preference. Pharmacol Biochem Behav 179: 9-21.

4. Facciol, A., Tran, S. and Gerlai, R. (2017). Re-examining the factors affecting choice in the lightdark preference test in zebrafish. Behav Brain Res 327: 21-28.

5. Gerlai, R., Lahav, M., Guo, S. and Rosenthal, A. (2000). Drinks like a fish: zebra fish (Danio rerio) as a behavior genetic model to study alcohol effects. Pharmacol Biochem Behav 67(4): 773-782.

6. Hascoët, M., Bourin, M., Nic Dhonnchadha, B. A. (2001). The mouse light-dark paradigm: a review. Prog Neuropsychopharmacol Biol Psychiatry 25(1): 141-166.

7. Maximino, C., de Brito, T. M., de Moraes, F. D., de Oliveira, F. V. C., Taccolini, I. B., Pereira, P. M., Colmanetti, R., Lozano, R., Gazolla, R. A., Tenório, R., de Lacerda, R. I. T., Rodrigues, S. T. K., Lameirão, S. V., Pontes, A. A. A., Romão, C. F., Prado, V. M. and Gouveia Jr., A. (2007). A 
comparative analysis of the preference for dark environments in five teleosts. Int $\mathrm{J}$ Comp Psychol 20(4): 351-367.

8. Maximino, C., de Brito, T. M., Dias, C. A. G. M., Gouveia, A., Morato, S. (2010). $\underline{\text { Scototaxis as }}$ anxiety-like behaviour in fish. Nat Protoc 5(2): 209-216.

9. Serra, E. L., Medalha, C. C. and Mattioli, R. (1999). Natural preference of zebrafish (Danio rerio) for a dark environment. Braz J Med Biol Res 32(12): 1551-1553.

10. Sousa, N., Almeida, O. F. X., Wotjak, C. T. (2006). A hitchhiker's guide to behavioural analysis in laboratory rodents. Genes Brain Behav 5: 5-24. 